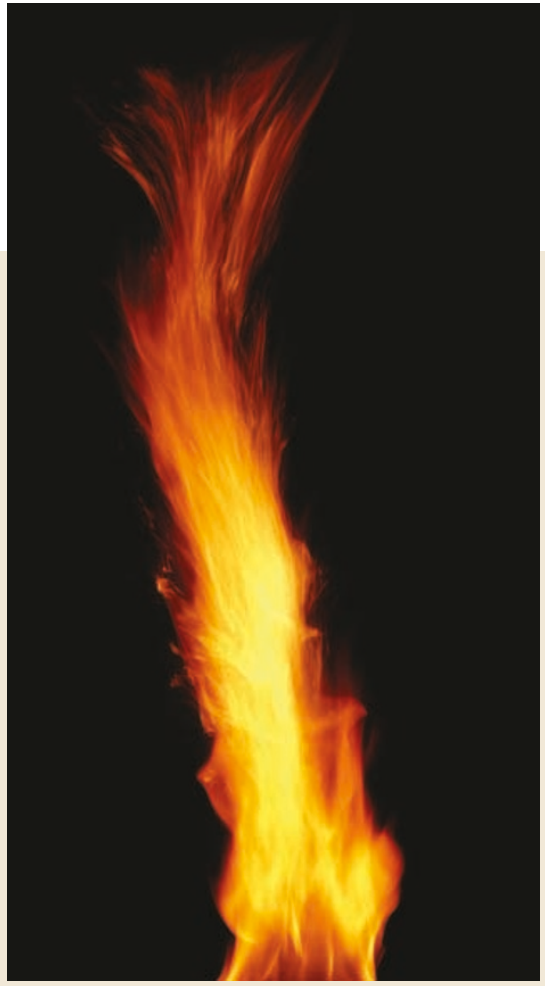

Manipulation of the process by which the body burns fat has long been a focus of efforts to treat obesity, but the underlying molecular pathways are not well understood. A recent paper published in Cell sheds light on this situation by showing that a ligand-activated transcription factor known as peroxisome-proliferator-activated receptor- $\delta$ (PPAR- $\delta$ ) stimulates fat burning, which indicates that drugs that activate PPAR- $\delta$ might have potential for treating obesity.

Although two other PPARs - PPAR- $\alpha$ and PPAR $-\gamma$ - are known to be important in fat metabolism, the role of PPAR- $\delta$ was unclear, and so Ronald Evans and colleagues set out to investigate its activity by using genetically modified mice. Selective expression of an activated form of PPAR- $\delta$ in adipose tissue resulted in mice with a lean phenotype,

although their food intake on a standard diet was normal. And when these mice were fed a high-fat diet, weight gain and lipid accumulation were markedly reduced compared with control mice. Further experiments with $L e p r^{\mathrm{d} b / \mathrm{db}}$ mice, which are predisposed to obesity, showed that expression of activated PPAR- $\delta$ reverses the obesity phenotype, and also that short-term treatment with a PPAR- $\delta$ agonist markedly depletes lipid accumulation.

All the mouse data are consistent with the idea that PPAR- $\delta$ activation upregulates fatty-acid combustion. But what is the molecular basis of this effect? To clarify this, the authors monitored the expression of genes involved in fatty acid metabolism, and found that PPAR- $\delta$ activation specifically induces the expression of genes required for fatty-acid oxidation and energy dissipation.

Overall, the authors' findings indicate that PPAR- $\delta$ is a key regulator of fat burning. PPAR- $\delta$ agonists might be particularly promising drug candidates for treating obesity as they can protect the body against both genetic and dietary obesity, and, furthermore, PPAR- $\gamma$ - which is closely related to PPAR- $\delta$ - is already validated as the target of antidiabetes drugs of the thiazolidinedione class.

(2) References and links

Peter Kirkpatrick

ORIGINAL RESEARCH PAPER Wang, Y.-X. et al.

Peroxisome-proliferator-activated receptor $\delta$ activates fat metabolism to prevent obesity. Cell 113, 159-170 (2003)

\title{
Overcoming antifolate drug resistance
}

Early medicinal chemists found that drugs developed from the structures of vitamins and nucleotides, such as the 2,4-diaminopyrimidine drug class, had potent and selective antiproliferative activity against malaria, and acted by blocking folate synthesis. Although Plasmodium falciparum drug resistance is a problem, antifolates, such as pyrimethamine (Pyr) and proguanil, have long been used clinically to treat malaria. The molecular basis of parasite resistance to these drugs arises from mutations in the parasite's bifunctional enzyme dihydrofolate reductase-thymidylate synthase (PfDHFR-TS). Now, in the May issue of Nature Structural Biology, Yuthavong and colleagues present crystal structures of wild-type, double-mutant and quadruple-mutant forms of PfDHFR-TS, in complex with different inhibitors.

PfDHFR-TS catalyses sequential reactions in the thymidylate cycle, and its inhibition prevents dTMP production and DNA synthesis. Unlike bacteria or higher eukaryotes that have separate
DHFR and TS proteins, the protozoan enzymes are joined as a bifunctional protein. The new structures reveal that, compared with host sequences, the active site of $P$. falciparum DHFR is more divergent than that of TS, and this feature facilitates mutations that compromise Pyr binding. The structures showed how a new, more flexible, antifolate - the triazine WR99210 - binds both wild-type and mutant forms at nanomolar concentrations.

The bifunctionality of the parasite enzyme indicates that the product from the TS step is rapidly moved to the DHFR active site, a phenomenon referred to as substrate channelling. Such channelling gives rise to more efficient overall catalysis than that of two separate monofunctional enzymes. However, the structures reveal that the distance between the active sites of TS and DHFR is too great to allow for simple channelling, but it is possible that there are two positively charged grooves on the surface

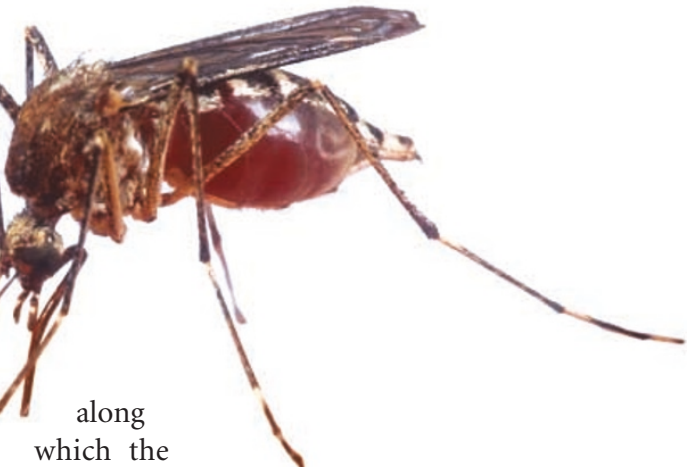

which the

DHFR substrate

might be channelled electrostatically.

Given the enormous toll of malaria as a disease, the PfDHFR-TS structure is of crucial importance. It will undoubtedly allow structure-function studies that will produce new Plasmodium-specific DHFR inhibitors to overcome antifolate resistance.

Melanie Brazil

\section{(Q) References and links}

ORIGINAL RESEARCH PAPER Yuvaniyama, J. et al. Insights into antifolate resistance from malarial DHFR-TS structrues. Nature Struct. Biol. 10, 357-365 (2003)

FURTHER READING Rathod, P. K. \& Philips, M. A. Prized malaria target nailed. Nature Struct. Biol. 10, 316-318 (2003) Hyde, J. E. Mechanisms of resistance of Plasmodium falciparum to antimalarial drugs. Microbes Infect. 4, 165-174 (2002) | Yuthavong, Y. Basis for antifolate action and resistance in malaria. Microbes Infect. 4, 175-182 (2002)

\section{WEB SITE}

Encyclopedia of Life Sciences: http://www.els.net Malaria 